Geophysical Research Abstracts,

Vol. 11, EGU2009-8613, 2009

EGU General Assembly 2009

(C) Author(s) 2009

\title{
A comparative study of Interior Layered Deposits on Mars
}

\author{
M. Sowe (1,2), L. H. Roach (3), E. Hauber (1), R. Jaumann (1,2), J. F. Mustard (3), and G. Neukum (2) \\ (1) German Aerospace Center (DLR), Institute of Planetary Research, Berlin, Germany (mariam.sowe@ dlr.de), (2) Institute of \\ Geological Sciences, Free University Berlin, Germany, (3) Dept. of Geological Sciences, Brown University, Providence, RI, \\ USA
}

Using information from high-resolution image (MOC, HiRISE), elevation (HRSC DTMs) and spectral data (TES, THEMIS, OMEGA, CRISM) a comparative study of Interior Layered Deposits (ILDs) located in the eastern Valles Marineris and their adjacent chaotic regions is performed in order to ascertain possible correlations between ILDs. ILDs have been reported from several regions around Valles Marineris that are in strong association with chaotic regions, hydrated minerals and haematite.

This study shows that ILDs partly vary in terms of erosional shape, thickness, elevation, material competence and possibly mineralogy, but that their morphologies are mostly comparable. Two different types of morphology were ascertained on their tops, these in turn apparently correlate with their mineralogical properties.

The majority of ILDs that exhibit a protective but spectrally neutral cap rock (showing surface vugs, monadnocks, sharp crests) on their top are the ones that coevally exhibit hydrated sulphates in their lower unit, whereas the ones that show a heavily fluted and grooved top tend to be spectrally neutral on the whole. At the time, the two types differ in their albedo as well as in their state of weathering and erosion. However, there is no correlation with elevation, thickness, or competence that could reinforce the above correlation.

Stair-stepped morphologies observed suggest alternating strata of competent and less competent material. Thickly bedded lower units and thinly layered upper units can be observed. Moreover, the ILD material is highly consolidated, which is confirmed by meter-sized boulders and talus produced by steep scarps, which is visible on HiRISE images. Different hydration states of sulphates (mono- and polyhydrated) are present here and have been found on ILDs in the western Valles Marineris as well. Thus, post-formational humidity changes may be responsible for volume changes resulting in angular joints and rock fragmentation into boulders. Convoluted-like bedding on the cap rock may also confirm this observation as they indicate dehydration. Hydrated sulphates demonstrate sufficient water or humidity was present up to a certain elevation suggesting a warmer, more humid Mars and acidic conditions that potentially existed in the Hesperian. The maximum elevation of hydrated sulphates corresponds to Ganges Mensa (-500 $\mathrm{m}$ ) for the chasmata and Iani Chaos ILDs $(-3000 \mathrm{~m})$ for the eastern chaotic terrains. This in turn indicates ILDs are in parts sulphate bearing up to the top, so the original uppermost elevation that experienced hydration could be higher than measured. 